\title{
Les sanctions du licenciement illégitime en France et en Italie : des droits sécurisés ou incitatifs aux licenciements?
}

Andrea Allamprese et Raphael Dalmasso

\section{(2) OpenEdition}

Journals

Édition électronique

URL : https://journals.openedition.org/rdctss/1671

DOI : $10.4000 /$ rdctss. 1671

ISSN : 2262-9815

Éditeur

Centre de droit comparé du travail et de la sécurité sociale

Édition imprimée

Date de publication : 1 avril 2019

Pagination : 136-153

ISSN : 2117-4350

\section{Référence électronique}

Andrea Allamprese et Raphael Dalmasso, « Les sanctions du licenciement illégitime en France et en Italie : des droits sécurisés ou incitatifs aux licenciements ? », Revue de droit comparé du travail et de la sécurité sociale [En ligne], 1 | 2019, mis en ligne le 01 novembre 2021, consulté le 13 novembre 2021. URL : http://journals.openedition.org/rdctss/1671; DOI : https://doi.org/10.4000/rdctss.1671

\section{(c) $($ ) $(9)$}

Revue de droit comparé du travail et de la sécurité sociale est mise à disposition selon les termes de la Licence Creative Commons Attribution - Pas d'Utilisation Commerciale - Pas de Modification 4.0 International. 


\section{LES SANCTIONS DU LICENCIEMENT ILLÉGITIME EN FRANCE ET EN ITALIE : DES DROITS SÉCURISÉS OU INCITATIFS AUX LICENCIEMENTS ?}

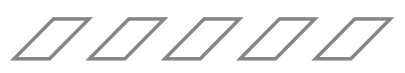

\section{RÉSUMÉ}

Cette contribution a pour objet d'étudier les récentes législations françaises et italiennes portant sur les licenciements, leur contrôle, et leur sanction en cas de motif illégitime. Les deux droits ont, entre 2012 et 2017, fait l'objet de profondes réformes qui commencent à produire leurs effets. Dans les deux droits, les législateurs ont justifié le changement par l'exigence d'une " sécurisation » des licenciements. L'étude prouve, outre le caractère ambivalent de ce terme, que l'objectif de sécurisation n'est pas atteint, pour aucune des parties.

MOTS CLÉS : Licenciement, régime de sanction, sécurisation.

\section{ABSTRACT}

This contribution sets out to study recent French and Italian legislation on redundancies, their monitoring and sanctions in the event of an improper motive. Between 2012 and 2017, these two legal systems were subject to major reforms which are now beginning to show their effects. In both systems, the justification for this change made by legislators was that it would make redundancies more "secure". This study proves, besides the ambivalent nature of this term, that the goal of security has not been achieved for either party.

KEYWORDS : Redundancies, Sanction System, Security. 
es droits du travail français et italien ont fait l'objet de profondes réformes entre 2012 et 2017', ayant provoqué, selon certains auteurs, un "changement de paradigme"2 concernant leur finalité, ceux-ci visant dorénavant, notamment, un objectif de sécurisation ${ }^{3}$ des relations de travail. Cet objectif est parfois présenté comme neutre, c'est-à-dire bénéficiant à la fois aux employeurs et aux salariés ${ }^{4}$, et consensuel, personne ne pouvant souhaiter vivre dans l'insécurité, a fortiori juridique. Nous proposons, dans cette recherche, d'étudier un point particulier, névralgique, des réformes françaises et italiennes, dans lequel l'objectif de sécurisation a été, des deux côtés des Alpes, invoqué. Il s'agit des modes de réparation des licenciements illégitimes.

Par conséquent l'objectif de la recherche est de tenter de déterminer des tendances exprimées par les deux systèmes de droit du travail. Dans les moments de dépression économique comme celle que nous vivons actuellement, les institutions juridiques, les lois et les normes en général, construites pour des périodes de tranquillité sociale sont remises en question ou menacées par les transformations des économies en crise ${ }^{5}$.

A partir de la fin des années 1960, la France et l'Italie, à l'instar de la quasi-totalité des pays européens, se sont dotées d'un cadre normatif relatif aux licenciements. Ces législations avaient comme objectif principal de rendre ce choix de gestion, pour l'employeur, comme

1 En France, la Loi Travail n²016-1088 du 8 août 2016 et surtout les ordonnances «Macron » de 2017. En Italie, la Loi «Fornero » n 92/2012, la Loi de délégation n 183/2014 et les décrets législatifs de 2105.

2 A. Perulli, «L'idea di diritto del lavoro, oggi », in A. Perulli (dir.), L'idea di diritto del lavoro, oggi. In ricordo di Giorgio Ghezzi, Kluwer-Cedam, Milano, 2018, p. XLI. ; A. Perulli, « Un nouveau paradigme pour le droit du travail, entre néolibéralisme et néolabourisme », Revue de droit du travail, 2015, p. 732 ; A. Perulli, « Les ordonnances Macron et le Jobs Act: valeurs et fonctions dans le nouveau paradigme du droit du travail », Droit social, 2018 p. 86 ; T. Sachs, C. Wolmark, "Les réformes de 2017, quels principes de composition?», Revue de droit du travail, 2017, p. 1008.

3 Sur cette notion, voir T. Sachs, "Quand la sécurité juridique se perd dans l'analyse économique », Droit social, 2015, p. 1019. Le thème de la sécurité juridique est cependant plus ancien. voir, par exemple, le numéro spécial de Droit Social de juillet-août 2006: B. Teyssié, "Sur la sécurité juridique en droit du travail », p. 703 ; A. Mazeaud, « Le principe de sécurité juridique: I'antidote au poison de l'insécurité juridique?», p. 707 ; F. Favennec-Hery, " Sécurité juridique et actes des partenaires sociaux », p. 766.

4 Concernant les ordonnances Macron, voir le dossier de presse du ministère du travail, p. 19. http://travail-emploi.gouv.fr/IMG/pdf/dossier_de_presse_-_conference_de_presse_31082017.pdf

5 B. Veneziani, Stato e autonomia collettiva. Diritto sindacale italiano e comparato, Cacucci, Bari, 1992. Pour une analyse consacrée aux mutations du droit du licenciement même dans les pays les plus touchés par la crise du 2008 voir B. Palli, " Les reformes nationales de la justification du licenciement au prisme des standards européens et internationaux », Revue Droit Travail, 2018, p. 618. 
un choix ultime (extrema ratio) ${ }^{6}$, toutes les autres solutions possibles ayant été envisagées. Si les droits du licenciement français et italien n'ont jamais souhaité interdire les ruptures (contraire au principe fondamental de liberté du travail), les législations italiennes de $1966^{7}$ et $1970^{8}$ et françaises de $1973^{9}$ ont posé un cadre procédural assez strict pour l'employeur.

Les orientations françaises et italiennes de ces années fondatrices ont cependant, avec la même intention, été très différentes. En France, la Loi de 1973 a instauré une réelle procédure préalable au licenciement (constituée, en résumé, d'une convocation, d'un entretien oral et d'une lettre de licenciement écrite) et a privilégié la voie de la réparation pécuniaire en cas de licenciement sans cause réelle et sérieuse. En Italie, l'article 18 du Statut des travailleurs a mis l'accent sur les sanctions, avec un double régime, en cas de licenciement illégitime, de réintégration (pour les moyennes et grandes entreprises) ou d'indemnisation (pour les plus petites structures). Malgré ces différences, l'objectif, identique, était de faire réfléchir l'employeur avant qu'il ne se libère de sa relation contractuelle avec le salarié, quel qu'en soit le motif (économique ou personnel).

Les réformes françaises et italiennes des années 2010 sont revenues sur ces régimes de réparation des licenciements illégitimes, ou, pour prendre la terminologie française, sans cause réelle et sérieuse. Dans les deux pays, ces changements constituent, en soi, un évènement. En Italie, l'art. 18 de la Loi n 300/1970 était considéré comme un bastion de la dignité, liberté et sécurité des salariés issu de cette grande avancée que fut le Statut des travailleurs (issu d'une période intense de négociation collective à la fin des années 60), de nombreux gouvernements ayant échoué dans les années 2000, à vouloir le modifier ${ }^{10}$. En France, le législateur a également essuyé un échec en 2015 et 2016 avant de pouvoir, finalement, imposer une modification substantielle des modes de réparation des licenciements.

Les droits passés étant fondamentalement différents, il ne faut pas tomber dans le piège de trop vite assimiler les réformes françaises et italiennes. Si le contexte économicopolitique est le même, la transposition juridique n'est pas être identique ${ }^{11}$, ce qui rend la

6 Voir notamment les propos d'A. Lyon-Caen concernant la jurisprudence Samaritaine: A. Lyon-Caen, Dalloz., 1997 J.. p. 171: « La Cour de cassation (...) invite aussi à se souvenir que la signification d'une règle - les licenciements pour motif économique sont l'ultime mesure possible - se marque avant tout dans la force de la sanction dont sa violation est assortie ». En Italie, voir M. Napoli, La stabilità reale del rapporto di lavoro, Milan, 1980, s'appuyant sur l'article 2103du Code civil italien pour justifier l'idée du licenciement comme extrema ratio.

7 Loi n 604/1966 du 15 juillet 1966.

8 Loi n 300/1970 du 20 mai 1970, communément appelée «Statut des travailleurs »..

9 Loi nº $73-680$ du 13 juillet 1973.

10 Voir notamment le projet de Loi de délégation du gouvernement « Berlusconi » n 848-bis de 2002, art. 3, qui prévoyait la suppression du caractère obligatoire de la réintégration en cas de licenciement sans cause juste pour les salariés titulaires d'un contrat à durée indéterminée du fait de la transformation d'un Contrat à durée déterminée.

11 R. Dalmasso, «Flexibiliser par la procédure », Travail et Emploi, 2015, n 142, p. 55. 
comparaison riche d'enseignements, mais aussi de pièges ${ }^{12}$. Aussi, nous pouvons dire que les réformes française et italienne sont deux laboratoires de transformation du droit du travail avec des protocoles et des solutions adoptées différents, mais visant un même objectif, assigné par une certaine forme de science économique ${ }^{13}$ : la sécurisation des ruptures. La mise en perspective des différences et des similitudes sera établie en privilégiant cependant la place du droit italien (I) le lecteur français étant supposé mieux connaître le droit français. Ces sécurisations font cependant désormais l'objet de contestation juridique. Celles-ci seraient contraires aux normes supérieures, constitutionnelles, voire de droit international du travail (II).

\section{I - DES TENTATIVES DE SÉCURISATION DES RUPTURES}

Pour bien comprendre la portée des récentes modifications (B), il faut, succinctement, revenir sur les normes originelles prévoyant l'indemnisation des licenciements illégitimes en France et en Italie (A).

\section{A - L'ÉMERGENCE DE DISPOSITIFS DISSUASIFS DE LICENCIEMENT}

En France comme en Italie, le droit du licenciement, dans les années 1960-1970, a été construit pour dissuader les ruptures. Le droit antérieur, aussi bien au niveau des motifs que de la procédure, était en effet considéré comme trop permissif. Cependant, les choix procéduraux effectués par les deux pays sont différents.

\section{1 - Le Statut des travailleurs italien de 1970: les protections en cas} de licenciement illégal

En Italie, depuis ses origines, la procédure limitative du licenciement n'est pas la même pour toutes les entreprises. Une distinction est à faire suivant le nombre, plus ou moins important, de salariés employés dans l'entreprise, ce qui génère donc, des droits différents, l'enjeu étant l'application ou non de la sanction de réintégration en cas de licenciement illégitime.

L'art. 2118 du Code civil italien prévoit un droit de rupture ad nutum des contrats à durée indéterminée. La réforme de 1966 - en lien avec les articles 4 et 35 de la Constitution - pose le principe de la nécessaire justification des licenciements. Ce principe a été affirmé par la

12 Cf. B. Veneziani, "Stato e autonomia collettiva. Diritto sindacale italiano e comparato », op. cit. ; O. Khan Freund, "Sull'uso ed abuso del diritto comparato », Rivista trimestrale di diritto della procedura civile, 1975, n² 2, p. 796.

13 Nous nous référons bien sûr à l'école Labour law \& economics sur le coût des ruptures (firing cost) qui doit être certain. 
Loi $n^{\circ} 604 / 66^{14}$ et confirmé par celle $n^{\circ} 300 / 70$ (et par celle $n^{\circ} 108 / 90$, modifiant les deux précédentes). Il se matérialise par deux formes de protections:

a) la protection obligatoire ("tutela obbligatoria"), prévue à l'art. 8 de la Loi n 604 comporte, en cas de licenciement - notifié par un petit employeur ${ }^{15}$ - privé d'une cause juste, motif justifié subjectif ou motif justifié objectif, de réembaucher le travailleur ou de lui verser une indemnité d'au moins 2,5 mois de salaire et au plus six mois de salaires.

b) la protection réelle ("tutela reale"), concernant les employeurs de taille plus importante ${ }^{16}$. Cette protection était prévue par l'art. 18 de la Loi $n^{\circ} 300 / 70^{17}$, qui dans la version précédente à la Loi $n^{\circ}$ 92/2012, fixait - dans toutes les hypothèses de licenciement injustifié, inefficace (car, par exemple, affecté de vices de procédure), ou nul car discriminatoire - l'obligation de l'employeur de réintégrer le travailleur et de lui donner une indemnité correspondant au préjudice subi (correspondant aux salaires non versés pendant la période entre le jour du licenciement jusqu'à la réintégration, déduction faite des autres revenus perçus durant cette période, avec un plafond de cinq mois de salaires), et, pour le travailleur, la possibilité de renoncer à la réintégration et d'obtenir en lieu et place une indemnité supplémentaire équivalente à 15 mois de salaires.

Le choix normatif d'un " parallélisme de protection » (avec réintégration dans le système de stabilité réelle ${ }^{18}$ et réparation par des dommages et intérêts dans celui de stabilité obligatoire) n'a pas manqué d'être évaluée sous le prisme d'une possible violation des principes d'égalité. Les juges constitutionnels ont cependant toujours retenu comme raisonnable la disparité de régime induite entre les travailleurs des grandes et petites

14 L'art. 3 prévoit que, dans le cadre du contrat à durée indéterminée, le licenciement du travailleur ne peut intervenir que pour une cause juste (giusta causa) au sens de l'art. 2119 du Code civil ou pour un motif justifié (giustificato motivo) subjectif ou objectif.

15 La protection obligatoire s'appliquait dans tous les cas où la protection réelle n'est pas applicable, c'est-à-dire (au sens de l'art. 2, Loi n 108/90) aux employeurs occupant jusqu'à 15 salariés dans une unité productive (ou jusqu'à cinq pour les entreprises agricoles), ainsi qu'aux employeurs qui, en toute hypothèse, occupaient jusqu'à soixante salariés; au sens de l'art. 3 de la Loi n 108/90, en cas de licenciement discriminatoire la réintégration trouvait application quel que soit l'effectif. Il y avait également des dispositions spécifiques relatives à certains employeurs (qui exercent des activités à caractère politique, syndical, etc.), qui étaient exclus du champ d'application de la protection réelle. Certains salariés (travailleurs domestiques, dirigeants, travailleurs en période d'essai) restaient assujettis à la règle de la rupture ad nutum.

16 Au sens de l'ancien alinéa 1 de l'art. 18 du Statut de travailleurs, la protection réelle s'appliquait aux employeurs (entrepreneurs ou non) occupant plus de 15 salariés dans une unité productive (ou plus de 5 pour les entreprises agricoles), et, dans tous les cas, àceux employant plus de soixante salariés. Cette disposition est aujourd'hui remplacée par les alinéas 8 et 9 de l'art. 18, au contenu identique.

17 Voir M. D’Antona, "Tutela reale del posto di lavoro », Enciclopedia Giur. Treccani, XXXI, Rome, 1994.

18 Voir M. Napoli, « La stabilità reale del rapporto di lavoro », op. cit., p. 41. 
entreprises, fondant leur argumentation sur deux aspects: a) la nécessité de ne pas grever de coûts excessifs les entreprises de petite dimension ${ }^{19}$; b) le niveau élevé de confiance dans les relations de travail dans ces entreprises, rendant impossible la réintégration ${ }^{20}$.

L'alternative entre stabilité « réelle » et stabilité « obligatoire » a donc toujours eu, pour la Cour constitutionnelle italienne, une justification de mérite, un peu paternaliste, de nécessaire compatibilité avec l'environnement des entreprises ${ }^{21}$ Ce régime a été largement modifié, comme nous le verrons plus tard, par le décret législatif n²3/2015 (voir infra).

\section{2 - La loi française de 1973: le choix de l'indemnisation et non celui de la réintégration}

A partir de la fin des années 1960, et, a fortiori, du début des années 197022, le législateur français comme les partenaires sociaux prennent conscience que le droit du travail, et plus précisément le droit du licenciement, doit donner des éléments de réponse juridique face à la crise. Cela va fréquemment se traduire, selon le vocabulaire de l'époque, par la notion de sécurité de l'emploi ${ }^{23}$. Mais derrière cette expression, invoquée de toutes parts, se cache en réalité une controverse : faut-il concevoir des dispositifs juridiques protégeant réellement l'emploi ou se contenter de normes encadrant les ruptures? Malgré une forte doctrine favorable à une protection réelle et contraignante de l'emploi, le législateur de 1973 opte pour de «simples » sanctions financières.

Le droit français du licenciement du début des années 1970 est en effet essentiellement jurisprudentiel, peu précis, et laissant au salarié une charge importante de la preuve en cas de contestation des motifs de la rupture. Une réforme législative s'avère donc nécessaire. Très rapidement deux options opposées vont s'affronter : d'un côté, la doctrine la plus favorable aux salariés, aidée par la position de la plupart des syndicats ${ }^{24}$, estime qu'en cas de licenciement illégitime il faut réintégrer le salarié ; de l'autre côté, une doctrine plus libérale, ou plus modérée, souhaite juste instaurer un dédommagement, sans remettre en cause la rupture.

19 Cour const. 6 mars 1974, n 55 ; Cour const., 14 avril 1969, n 81 ; et pour la légitimité de la distinction entre entreprises industrielles et agricoles, Cour const., 19 juin 1975, n 152.

20 Cour const., 8 janvier 1986, n² 2 ; plus en général Cour const., 23 février 1996, n 44.

21 Concernant la catégorie des dirigeants, l'exclusion de la protection réelle a été justifiée en se référant aux caractéristiques particulières des modes d'exécution de la relation de travail Cf. Cour const., 26 octobre 1992, n 404 ; Cour const., 7 mai 1975, n 101 ; Cour const., 6 juillet 1972, n 121.

22 On passe de 439.000 demandeurs d'emploi en mars 1974 à 755.000 en mars 1975.

23 Voir notamment l'accord national interprofessionnel du 10 février 1969 portant sur «la sécurité de l'emploi ». Sur ce texte, G. Battu, «Analyse de l'accord national interprofessionnel du 10 février 1969, modifié par l'avenant du 21 novembre 1974 », Droit social, juin 1975, numéro spécial, p. 27.

24 Les grandes centrales syndicales CGT, CFDT, CGT-FO, ainsi que le syndicat de la magistrature, sont en accord sur ce combat. Voir notamment, E. Maire (secrétaire général de la CFDT), Le Monde 22 août 1972, p. 14: «La Loi devra intervenir. Elle devra retenir les principes suivants: (...) preuve d'un motif justifié à la charge de l'employeur ; à défaut de cette preuve, impossibilité ou nullité du licenciement, le travailleur pouvant choisir entre la réintégration ou une véritable indemnisation ». 
Dans un article de 1973, F. Naudé, exprimant la position de la CFDT, défend fortement, avant le vote de la réforme du droit des licenciements, l'instauration d'une réelle protection de l'emploi en cas de licenciement abusif, avec comme sanction la réintégration du salarié: "Alors que le principe de la nullité du licenciement prononcé en violation des dispositions légales n'est plus discuté dans un certain nombre de pays (Allemagne, Italie), ainsi que le principe de la réintégration qui en découle, le projet de loi ne prévoit la réintégration que si le patron et le travailleur l'acceptent. L'idée dominante reste donc celle selon laquelle la réintégration n'est pas possible contre la volonté de l'employeur. La réintégration serait la seule mesure qui puisse réparer le préjudice résultant pour l'employeur de la privation de son emploi et reconnaître son droit au travail $\aleph^{25}$.

La Loi du 13 juillet 1973 impose à l'employeur de motiver le licenciement ${ }^{26}$, et au juge de contrôler le caractère "réel et sérieux $»^{27}$ desdits motifs ${ }^{28}$. Dans cette Loi, le choix opéré est cependant celui d'une indemnisation du salarié licencié sans cause réelle et sérieuse, principe figurant jusqu'à aujourd'hui dans l'article L.1235-3 du Code du travail29, au détriment de la logique de réintégration. G. Lyon-Caen et M.-C. Bonnetête, commentent à l'époque de manière critique ce choix: « La Loi du 13 juillet 1973 laisse donc subsister le droit unilatéral et préalable de licencier. Elle ne pouvait probablement pas, dans la conjoncture où elle est intervenue, faire autre chose. Loi modeste, de capitulation devant les faits, plutôt que de réforme. Il en est également ainsi pour la réintégration du salarié en cas de licenciement irrégulier (...). Alors que plusieurs juridictions de référés au cas le licenciement est non seulement fautif mais prononcé en violation directe de la Loi n'hésitaient plus à ordonner la réintégration, la Loi fait marche arrière et rend la réintégration toujours facultative pour l'employeur. La Loi est donc de fermeture, non d'ouverture $»^{30}$.

Le législateur de 1973 a certainement été sensible aux arguments d'une doctrine invoquant déjà le risque d'un effet pervers d'une trop grande protection des salariés sur les décisions d'embaucher. A. Sauvy indique ainsi, avec un argumentaire d'une étonnante actualité: "L'affaire LIP et bien d'autres ont montré combien les salariés étaient devenus sensibles à la crainte d'un licenciement, particulièrement les cadres, et combien ils souhaiteraient la sécurité de ce côté. (...) Supposons que, sous la pression des syndicats

25 F. Naudé, "Réflexion à propos du projet de loi modifiant le droit du licenciement », Droit social, 1973, p. 148. Voir aussi, J.-J. Dupeyroux, Le Monde, 4 juillet 1972, p. 12.

26 Art. L.1232-6 Code du travail (Anc. art. L.122-14-2, alinéa 1).

27 Art. L.1232-1 Code du travail (Anc. art. L.122-14-3, alinéa 1, phrase 1). Voir aussi art. L.1235-1 Code du travail (Anc. art. L.122-14-3, alinéa 1, phrase 1, et al. 2).

28 L'art. 24q de la Loi du 13 juillet 1973 précisait cependant expressément ne pas être applicable " aux licenciements collectifs pour motif économique ». Le législateur estimait que l'accord interprofessionnel sur la sécurité de l'emploi du 10 février 1969 accordait des protections suffisantes aux salariés.

29 Cet article indique encore aujourd'hui qu'en cas de licenciement sans cause réelle et sérieuse «le juge peut proposer la réintégration », mais l'employeur peut s'y opposer.

30 G. Lyon-Caen, M.-C. Bonnetete, "La réforme du licenciement à travers la loi du 13 juillet 1973 ", Droit social. 1973, pp. 495-496. 
et aussi de l'opinion, les pouvoirs publics décident d'interdire les licenciements, ou de les rendre très difficiles. Les entreprises ne recruteront désormais qu'avec prudence, attitude qui retombera cette fois encore sur les jeunes $»^{31}$.

Dès lors, plus qu'une loi protectrice des emplois, la Loi de 1973 sur le caractère réel et sérieux des licenciements s'inscrit dans une logique d'encadrement, assez strict, des ruptures, moyennant, le cas échéant, indemnisation pécuniaire des salariés.

Les droits français et italien de la fin des années 1960 et du début des années 1970 optent donc pour des choix différents en matière de réparation des licenciements illégitimes. Cependant, bien que cette expression n'ait pas encore été employée à l'époque, les deux régimes ont pour objectif commun de dissuader l'employeur de procéder à un licenciement, par le biais de la motivation, mais, surtout, par la sanction de l'absence de motivation. Réintégrer ou indemniser, le but est le même: outre dédommager de manière complète le préjudice subi par le salarié, il s'agit d'inciter l'employeur à n'utiliser le licenciement que comme choix extrema ratio. L'Italie, avec la protection réelle imposant réintégration, a cependant été plus loin dans l'idée de la nécessaire réparation intégrale du dommage subi par le salarié.

\section{B - LA VOLONTÉ DE LIMITER ET DE PRÉVOIR L'AMPLEUR DE LA RÉPARATION}

Une même critique va être adressée aux droits français et italien concernant la réparation des licenciements, à l'orée des années 2000. Les sanctions sont trop fortes, et surtout incertaines, dissuadant l'employeur de procéder à des embauches. Aussi, les réformes menées en Italie et en France entre 2012 et 2017 se justifient, d'un point de vue juridique, essentiellement par une volonté de sécuriser les ruptures. Bien entendu, partant de droits différents, les évolutions française et italienne sont différentes. Ici, la place du droit italien sera privilégiée, le droit français contemporain étant supposé connu.

\section{1 - Les réformes italiennes depuis 2012}

Deux séries de réformes vont voir le jour en Italie. La première en 2012 (réforme dite «Fornero ») (a) et la deuxième en 2014-2015 (réforme « Renzi ») (b).

\section{a - La «Loi Fornero »: cantonnement de possibilités de réintégration et promotion d'un barème d'indemnisation}

La Loi italienne n 92 de 2012 (dite «Fornero ») a innové en ce qui concerne la sanction d'un licenciement illégal, en modifiant, outre la substance, le titre de l'art. 18 de la Loi $n^{\circ} 300 / 70$, remplacé par le texte suivant: «Protection du travailleur en cas de licenciement illégitime » (art. 1er, alinéa 42, point a).

En application des trois premiers alinéas du «nouvel » art. 18 (qui continue de ne s'appliquer qu'aux travailleurs déjà embauchés en contrat à durée indéterminée à la date du 7 mars 2015, date d'entrée en vigueur du décret législatif $n^{\circ} 23 / 2015$ ), la protection réelle trouve application générale, quels que soient les effectifs de l'employeur, pour

31 A. Sauvy, « Dérive et accident », Droit social, 1973, p. 490. 
tout licenciement discriminatoire, ou encore « imputable à d'autres cas de nullité prévus par la loi ou déterminés par un motif illicite décisif » au sens de l'art. 1345 du Code civil, indépendamment du motifformel donné par l'employeur; cette sanction affecte également un licenciement inefficace car prononcé oralement.

Pour les employeurs qui emploient plus de quinze salariés dans l'unité de production ou plus de 60 travailleurs au total, la sanction de réintégration (quoique « atténuée » par le fait que l'indemnité correspondante ne peut dépasser le plafond de douze mois de salaire) est également prévue en cas de licenciement pour motif justifié objectif, lorsque le fait avancé par l'employeur est manifestement non fondé ("manifesta insussistenza del fatto" ex art. 18, alinéa 7), et en cas de violation des critères de sélection des travailleurs à licencier collectivement (art. 5, alinéa 3, troisième phrase, Loi $n^{\circ}$ 223/92) ; la même sanction est également prévue en cas de licenciement disciplinaire injustifié pour « inexistence du fait contesté »(art. 18, alinéa 4) (voir infra).

Compte tenu de ce qui précède, ceux qui figurent à l'alinéa 1 de l'art. 18 (en premier lieu, le licenciement discriminatoire) représentent les hypothèses principales de licenciement illégitime sanctionnés par la réintégration. C'est pourquoi à partir de 2012, nous avons été témoin d'une tendance à réclamer à l'avance devant les tribunaux - la nullité du licenciement ci-dessus, malgré les difficultés de charge de la preuveici supportées - par le travailleur, afin d'attirer dans le cadre de la réintégration la grande majorité des litiges relatifs au caractère illégitime des licenciements pour raisons disciplinaires ou économiques. Le cas le plus récurrent et le plus difficile à prouver n'est pas celui de la discrimination au sens strict (politique syndicale, raciale ou religieuse), mais celui du licenciement-rétorsion, et notamment en représailles à lıexercice dıun droit, constitutionnel ou même simplement inhérent au contrat de travail ${ }^{32}$. La Loi n92/2012 n'ignore pas ce problème: elle prévoit la déclaration de nullité/réintégration (aussi) du licenciement déterminé par un motif illicite décisif au sens de l'art. 1345 du Code civil, même si le texte reste assez générique, et avec le défaut de laisser la place à une interprétation restrictive selon laquelle le motif, pour être réellement décisif, doit aussi être « unique $»^{33}$.

32 On pense, par exemple, au salarié licencié pour avoir engagé une action en justice pour le rappel d'heures non payées (Trib. Parma, 1 février 2018, Rivista giuridica del lavoro, 2018, II, p. 385), ou pour avoir témoigné e en justice dans un sens défavorable à l'employeur (Trib. Prato, 14 novembre 2017, Rivista giuridica del lavoro, 2018, II, p. 416), ou pour avoir dénoncé des violations graves du code de déontologie par un supérieur (Trib. Florence, 13 septembre 2018, inédit).

33 Voir Cass., 5 avril 2016, n 6575, Rivista italiana di diritto del lavoro, 2016, II, p. 714, qui a précisé le rapport entre le licenciement discriminatoire et le licenciement pour motif illicite. 
Le licenciement pour motif justifieésubjectifest réglementé par les alinéas 4 et 5 de l'art. 18. La législation est fondée sur une distinction entre les différents vices qui peuvent rendre le licenciement disciplinaire illégitime: une distinction est faite entre certains vices qui peuvent donner lieu à l'annulation du licenciement et à la réintégration (licenciement injustifié pour « inexistence du fait contesté » ex art. 18, alinéa $4^{34}$ ) et tous les " autres» qui laissent le licenciement injustifié efficace et ne donnent lieu qu'à versement d'une indemnité. En particulier, selon l'alinéa 5 de l'art. 18, dans les « autres hypothèses »dans lesquelles le juge constate que les conditions requises du motif justifié subjectif ou de la cause juste ne sont pas réunies, il déclare la rupture du contrat de travail avec effet à la date du licenciement et condamne l'employeur à verser une indemnité comprise entre un minimum de douze et un maximum de 24 mois du dernier salaire, calculée en fonction d'un certain nombre de critères (ancienneté, nombre de salariés, activité économique, comportement des parties) ${ }^{35}$.

Enfin, celle du licenciement pour motif justifié objectif était la question la plus débattue au moment de l'approbation de la Loi «Fornero », car l'intention initiale du gouvernement "Monti » était de prévoir uniquement des dommages et intérêts, et non la réintégration, en cas d'illégitimité du licenciement. Dans le texte de l'alinéa 7 de l'art. 18, la possibilité de réintégration (version " atténuée » de l'art. 18, alinéa 4) a été réintroduite. Au sens de cette règle, le juge " peut » attribuer au salarié la protection réelle (avec réintégration) en cas $d^{\prime}$ " inexistence manifeste » du «fait à la base du licenciement pour motif justifié objectif» (2ème phrase); dans les « autres hypothèses, où il constate que les conditions du motif justifié objectif ne sont pas réunies », le juge appliquera la protection obligatoire.

Par conséquent, clarifier la signification de certaines expressions - en particulier le concept de «fait à la base du licenciement » et la notion d' « inexistence manifeste »signifie tracer une frontière entre réintégration et indemnisation en cas d'absence de motif objectif de licenciement.

La Cour de Cassation italienne a pris position pour clarifier ces points. En particulier, les juges italiens ont d'abord identifié le « fait » avec un concept juridique et précisément avec le motif justifié objectif de type économique fixé par l'art. 3 de la loi n 604/66, ce motif étant composé de trois éléments: la réorganisation effective (de quelque type que ce soit: suppression de poste, innovation technologique, etc.), un lien causal entre cette réorganisation et la position du salarié licencié, et l'obligation de reclasser le travailleur ${ }^{36}$. Une fois le "fait » identifié avec le concept de motif justifié objectif de type économique,

34 La Cour de Cassation italienne a clarifié la notion de fait inexistant. Il peut être compris comme un fait matériellement existant mais qui ne caractérise pas un manquement du contrat, donc privé d'illicéité. Dans cette hypothèse, il faut appliquer la protection réelle avec réintégration. Dans ce sens Cass. 10 mai 2018, n 11322 ; Cass., 26 mai 2017, n 13383 ; Cass. 25 mai 2017, n 13178 ; Cass. 20 septembre 2016, n 18418 ; Cass. 13 octobre 2015, n²0540. La thèse du «fait juridique » est désormais accueilli par la jurisprudence italienne majoritaire, voir Cass. 3 septembre 2018, n 21569, Rivista giuridica del lavoro, $\mathrm{n}^{\circ} 1 / 2019$.

35 La Cour de Cassation italienne (Sections Unies, arrêt n 30985 du 27 décembre 2017) a ramené les cas de violation du principe de l'opportunité de la contestation disciplinaire (qui n'est qu'un des vices les plus courants de la procédure ex art. 7 de la Loi n³00/70) aux « autres hypothèses » d'illégitimité du licenciement (donc à un vice substantiel). Dans cette hypothèse, le salarié a droit à une indemnité (art. 18, alinéa 5).

36 Pour l'inclusion de l'obligation de reclasser le salarié dans la notion de « fait » voir Cass. 22 octobre $2018, n^{\circ} 26675$. 
les juges ont indiqué que le manque de preuve de la part de l'employeur d'un seul des trois éléments détermine l'« inexistence manifeste du fait » en référence à l'art. 18, alinéa 7, Loi $n^{\circ} 300 / 70^{37}$.

\section{b - La « Loi Renzi »}

La réforme de 2012 n'a pas été jugée suffisante par le gouvernement italien « Renzi », qui l'a jugée trop rigide et susceptible de nuire à l'emploi. L'élément de rigidité a été identifié à la fois dans le fait que la loi laisse subsister la réintégration en cas de licenciement pour motif justifié objectif et dans le fait qu'elle ne garantit pas à l'employeur la certitude et la prévisibilité du coût du licenciement, laissant au juge le soin de déterminer le montant des indemnités à verser au salarié.

Par le décret législatif n 23/2015 (modifié par l'art. 3, alinéa 1, du décret-loi n 87/2018, converti en loi n 96/2018), le gouvernement «Renzi » a donc voulu combler cette «lacune»: d'une part, en réduisant encore les cas dans lesquels il est possible d'obtenir la réintégration ; d'autre part, en abaissant le montant des indemnités pour licenciement abusif et, surtout, en éliminant l'incertitude concernant leur quantification (et en limitant ainsi la possibilité pour le juge de moduler la sanction en fonction du préjudice concrètement subi).

En application du critère de délégation fixé par la Loi $n^{\circ} 183$ de $2014^{38}$, le décret législatif $n^{\circ} 23 / 2015$ sur le contrat de travail à durée indéterminée "à protections croissantes » («a tutele crescenti ») définit tout d'abord son champ d'application par référence aux «travailleurs qui ont le statut de ouvriers, employés ou cadres, engagés en vertu d'un contrat de travail à durée indéterminée à compter de la date d'entrée en vigueur » du décret ( 7 mars 2015) (art. 1er, alinéa 1), y compris - conformément à l'art. 9, alinéa 2 - ceux employés par des employeurs qui ne sont pas des entrepreneurs «qui exercent des activités à caractère politique, syndical, culturel ou religieux, sans but lucratif »; il semble donc clair qu'il existe une double limite: I'exclusion explicite

37 Dans ce sens, voir entre autres, Cass. 2 mai 2018, n 10435, Rivista giuridica del lavoro, 2018, II, p. 459. Les juges ont de plus retenu que l'expression « inexistence manifeste » du faitprend un sens sur le plan de la preuve. Ainsi le juge du fond « doit vérifier si l'inexistence même d'un seul des éléments constitutifs du licenciement est manifeste ou évidente » ; le législateur " a voulu limiter à des hypothèses résiduelles le droit à la réintégration » et donc le concept d'« inexistence manifeste » doit être référé " à une évidente et facilement vérifiable (...) absence des hypothèses justificatives du licenciement permettant d'apprécier que la rupture est clairement un prétexte ». Selon cette interprétation, l'art. 18, alinéa 7, aurait gradué les conséquences en matière de sanction d'un licenciement illégitime ; seul le licenciement "totalement » privé de motif justifié objectif ( «licenciement prétexte ») doit faire l'objet d'une réintégration ; à l'inverse, le licenciement " simplement » privé de motif justifié objectif implique la protection obligatoire de l'art. 18, alinéa 5 (indemnisation entre douze et 24 mois du dernier salaire). Pour une critique de cette position voir M.T. Carinci, « Licenziamento per gmo e repechage », Rivista giuridica del lavoro, 2018, II, p. 466.

38 Par l'art. 1, alinéa 7, point c), de cette Loi, le gouvernement a reçu mandat pour publier une réforme de la réglementation des licenciements sur la base des principes suivants: " prévoir, pour les nouvelles recrues, un contrat à durée indéterminée assorti de garanties croissantes en matière d'ancienneté, excluant pour les licenciements économiques la possibilité de réintégrer le salarié, prévoyant une indemnisation économique certaine et croissante en fonction de l'ancienneté et limitant le droit de réintégration aux licenciements nuls et de nul effet et discriminatoires ainsi qu'aux cas spécifiques de licenciement disciplinaire injustifié et prévoyant des délais précis pour faire appel contre un licenciement ». 
des travailleurs déjà employés sous contrat permanent ( avant le 7 mars 2015) et des dirigeants; l'exclusion implicite des employés publics privatisés.

Deuxièmement, le décret législatif $n^{\circ} 23 / 2015$, toujours en application de la loi n 183/2014, établit à l'art. 3, alinéa 1, pour les travailleurs nouvellement embauchés, en cas de licenciement considéré comme illégitime parce qu'il manque les conditions de validité de la raison objective justifiée ou de la raison subjective justifiée ou de la juste cause - qui représentent la prévalence des cas soumis aux tribunaux -, un modèle général de protection de type indemnisation, certain dans son montant, progressive dans son augmentation basée exclusivement sur l'ancienneté du salarié et soumise à un plafond dans son montant maximal (répondant ainsi au critère prévu par l'art. 1, alinéa 7, point c) : « une indemnisation certaine et croissante en fonction de l'ancienneté »).

Le modèle général de protection visé à l'art. 3, alinéa 1, se distingue - par son montant fixe et progressif - en fonction des effectifs de l'entreprise. II prévoit désormais, depuis les réformes du gouvernement "Conte » (décret-loi n 87/2018), un montant minimum $d^{\prime}$ indemnisation, respectivement, de six mois de salaire ${ }^{39}$ pour les employeurs comptant plus de 15 salariés dans l'unité de production individuelle et deux mois de salaire pour les autres employeurs - majoré, dans la limite de deux mois du dernier salaire (de référence pour le calcul de l'indemnité de départ) pour chaque année de service, dans le premier cas, et d'un mois de salaire pour chaque année de service, dans le second cas. Le montant maximal de l'indemnisation est désormais de 36 mois pour les salariés des employeurs comptant plus de 15 salariés dans l'unité de production individuelle et de six mois pour les autres ${ }^{40}$.

\section{C - LA RÉFORME FRANÇAISE DE 2017 : BARÈME ET PLAFOND AU NOM DE LA SÉCURITÉ}

En France, après plusieurs tentatives avortées ${ }^{41}$, les ordonnances «Macron ${ }^{42}$ " ont finalement pu imposer une forme de barémisation et de plafonnement des indemnités de licenciement sans cause réelle et sérieuse. II n'apparaît pas nécessaire de revenir

39 Le décret-loi n 87/2018 a modifié il régime de sanction de l'art. 3, alinéa 1, augmentant le montant de l'indemnité en cas de licenciement illégitime: avec désormais un minimum de six mensualités (au lieu de quatre) et un maximum de 36 mensualités (au lieu de 24).

40 II existe quelques rares exceptions. La réintégration reste la règle dans les hypothèses prévues à l'art. 2 du décret législatif n²3/2015 (licenciement nul), et dans celle prévue à l'art. 3 al. 2, du même décret, en cas de licenciement pour motif justifié subjectif dans lequel le juge constate l'inexistence du fait matériel allégué, en dehors de toute évaluation relative à la proportionnalité du licenciement.

41 Voir notamment la première tentative d'instaurer un barème, prévu par la loi « Macron " n²015990, censuré par la décision du Conseil constitutionnel n² 2015-715 du 5 août 2015 (AJDA 2015, 1570). Voir notamment D. Baugard, " Le plafonnement de l'indemnisation des licenciements injustifiés ne peut pas varier selon les effectifs des entreprises », Droit social, 2015, p. 803 ; T. Sachs, "Quand la sécurité juridique se perd dans l'analyse économique », Droit social, 2015, p. 1019. V. aussi J. Mouly, « Le plafonnement des indemnités de licenciement injustifié devant le Comité européen des droits sociaux », Droit social, 2017, p. 745.

42 Ordonnances $n^{\circ} 1385,1386,1387,1388$ e 1389 du 22 septembre 2017 et celle $n^{\circ} 1718$ du 20 décembre 2017, adoptées suite à la loi d'habilitation n²017-1340 du 15 septembre 2017 et ratifiées par la loi n²018-217 du 29 mars 2018. 
ici sur ces dispositions largement étudiées dans les revues juridiques françaises ${ }^{43}$, et figurant aux articles L.1235-3 et suivant du Code du travail, qui définissent, en fonction de l'ancienneté du salarié et de la taille de l'entreprise, des planchers, et, surtout, des plafonds d'indemnisation.

Mentionnons cependant que le titre du chapitre II de l'ordonnance $n^{\circ}$ 2017-1387 montre l'intention du législateur: il s'agit désormais de « réparer » le licenciement et non de dédommager le salarié de son préjudice, Selon F. Batard et M. Grévy ${ }^{44}$, «la sanction civile se dissout plus que jamais dans une «taxation » dont l'objet est de dissuader le salarié d'agir en justice ou, à tout le moins, de permettre à l'employeur d'anticiper et de provisionner exactement le montant auquel, le cas échéant, il sera condamné ».

Le lien avec la réforme "Renzi » de 2015 est ainsi étroit: dans les deux systèmes, le législateur a cherché à limiter l'indemnité de licenciement illégitime et à la rendre plus prévisible pour l'employeur. Sur le site servicepublic.fr, un simulateur en ligne est même dorénavant disponible pour calculer, en fonction de l'ancienneté du salarié et des effectifs de l'entreprise, ce plancher et ce plafond possible d'indemnisation ${ }^{45}$. Notons que les plafonds français sont inférieurs à ceux fixés par le législateur italien.

Comme en Italie, de rares exceptions peuvent permettre d'échapper au plafond. De manière dérogatoire, le montant de l'indemnité prud'homale ne peut pas être inférieur aux salaires des six derniers mois lorsque le juge constate que le licenciement est nul parce qu'il est intervenu, notamment: en violation d'une liberté fondamentale, en lien avec des faits de harcèlement moral ou sexuel, en application d'une mesure discriminatoire ${ }^{46}$ ou à la suite d'une action en justice engagée par le salarié sur la base des dispositions réprimant les discriminations ${ }^{47}$.

\section{II - COMPATIBILITÉS PROBLÉMATIQUES DES LÉGISLATIONS FRANÇAISE ET ITALIENNE AVEC LES NORMES SUPÉRIEURES}

Ces « nouveaux » droits, plafonnant les indemnisations, sont censés sécuriser les ruptures. Cependant, ce faisant, le risque couru par les deux législations nationales est $d^{\prime}$ insuffisamment dissuader le recours au licenciement. La Cour constitutionnelle italienne a récemment remis en cause toute la pertinence de la réforme "Renzi » $(A)$, tandis que la compatibilité de ces législations avec des normes internationales est posée (B). Cependant, un enjeu particulier demeure, celui du contrôle judiciaire des motifs économiques (C).

43 Voir ainsi le numéro spécial de la Revue de droit du travail d'octobre 2017, et notamment P. Adam, "Libertés fondamentales et barémisation: la grande évasion », p. 643 ; F. Batard, M. Grévy, "Securitas omnia corrumpit», p. 663. i C. Percher, "Le plafonnement des indemnités de licenciement injustifié à l'aune de l'art. 24 de la Charte sociale européenne révisée », Revue de droit du travail, 2017, p. 726.

44 F. Batard, M. Grévy, « Securitas omnia corrumpit», op. cit.

45 https://www.service-public.fr/simulateur/calcul/bareme-indemnites-prudhomales

46 Cf. Cass. soc. 15.11.2017, n 16-14.281. Pour un commentaire M. Mercat-Bruns, « Le principe de non-discrimination en raison de l'âge ne constitue pas une liberté fondamentale ", Revue de droit du travail, 2018, p. 132.

47 Voir pour la liste complète art. L. 1235-3-1 Code du travail. 


\section{A - LA Cour constitutionnelle itAlienne et l'inconstitutionalité du Jobs ACT}

Un peu plus de quatre mois après l'entrée en vigueur du décret-loi n 87/2018, a été publié le jugement de la Cour constitutionnelle italienne $n^{\circ} 194$ de $2018^{48}$ statuant sur une question de constitutionnalité soulevée par le Tribunal de Rome concernant l'art. 3, alinéa 1, du décret $n^{\circ}$ 23/2015.

La Cour constitutionnelle a déclaré illégitime l'art. 3, alinéa 1, précité, dans la partie - non modifiée par le décret-loi n 87/2018 - qui prévoit un mécanisme de calcul automatique, fondé sur l'ancienneté du salarié, des dommages et intérêts causés par la rupture injustifiée.

Le premier motif de saisine de la Cour constitutionnelle portait sur la violation de l'article 3 de la Constitution italienne (principe d'égalité). En effet, selon les requérants, et le Tribunal de Rome, la loi instaurant deux systèmes juridiques différents en fonction de la date d'embauche des salariés portait atteinte au principe d'égalité. La disparité de traitement entre anciens et nouveaux embauchés était considérée comme privé de justification. Le Tribunal de Rome avait ainsi indiqué que « la date d'embauche apparaît comme une donnée accidentelle » et qu'aucun élément ne pouvait justifier une telle différence de traitement.

La Cour constitutionnelle a cependant refusé cette argumentation. Selon la Cour le critère de détermination de l'application dans le temps du décret législatif n 23/2015 «ne s'oppose pas au principe du « raisonnable» ("ragionevolezza »). La Cour estime que le but («scopo ») poursuivi par le législateur de renforcer les opportunités d'entrée dans le monde du travail permet de justifier un droit du licenciement différent entre anciens et nouveaux embauchés.

La Cour s'est ici limitée à l'examen du caractère apparemment raisonnable et non sur le fond. Elle a ainsi précisé qu'« il n'appartient pas à cette Cour d'entrer dans l'appréciation des résultats que la politique de l'emploi menée par le législateur cherche à obtenir ».

Le jugement d'illégitimité constitutionnelle s'est donc centré sur l'art. 3, alinéa 1, du décret législatif $n^{\circ} 23 / 2015$ (soit dans son texte originaire, soit dans le texte modifié par l'art. 3, alinéa 1, du décret-loi n 87/2018).

Dans l'affaire au principal devant le Tribunal de Rome, le licenciement infligé à la travailleuse plaignante - quelques mois après son embauche avec un contrat à protections croissantes - se basait sur la motivation suivante: «suite à des problématiques croissantes à caractère économique et de production ne permettant pas la poursuite régulière de la relation de travail, son activité professionnelle ne peut plus être fructueuse pourl'entreprise ». L'employeur avait en outre mentionné l'absence de reclassement possible, et licencié pour motif juridique objectif la salariée. Les juges du Tribunal de Rome ont considéré que la

48 Cour const. 8 novembre 2018, n 194, Dejure, p. 1 ss. Cf., notamment, V. Speziale, " La sentenza $\mathrm{n}^{\circ} 194$ del 2018 della Corte costituzionale sul contratto a tutele crescenti ", Rivista giuridica del lavoro, n 1/2019; S. Giubboni, « Il licenziamento del lavoratore con contratto "a tutele crescenti" dopo I'intervento della Corte costituzionale », Foro Italiano, n 1/2019; A. Perulli, « II valore del lavoro e la disciplina del licenziamento illegittimo ", Libro dell'anno del diritto Treccani, Rome, 2018, p. 339 ; M. T. Carinci, « La Corte costituzionale n. 194/2018 ridisegna le tutele economiche per il licenziamento individuale ingiustificato nel "Jobs Act", e oltre ", WP "Massimo D'Antona", n³78/2018; R. De Luca Tamajo, "La sentenza costituzionale 194 del 2018 sulla quantificazione dell'indennizzo per licenziamento illegittimo », Diritti Lavori Mercati, 2018, p. 633 ; P. Saracini, «Licenziamento ingiustificato: risarcimento e contenuto essenziale della tutela », op. cit., p. 643. 
motivation adoptée « est extrêmement générique et adaptable à n'importe quelle situation, et donc inapte à atteindre le but auquel devrait aboutir la charge de la motivation »; "face au caractère on ne peut plus général de la motivation adoptée (...) ce vice est le plus grave de ceux indiqués, à savoir l'absence des « conditions requises du licenciement pour motif justifié objectif ». Ils ont ajouté que si la salariée avait été embauchée avant le 7 mars 2015, elle aurait eu droit à la réintégration et à une indemnité de douze mois (ex art. 18, alinéa 7, L. $n^{\circ} 300 / 70$ ) ou - en application de l'alinéa 5 de l'art. 18 - une indemnisation comprise entre douze et vingt-quatre mois de salaires, et qu'elle n'a eu en l'espèce droit qu'à quatre mois de salaire. En définitive, il subsistait pour le Tribunal un vice de motivation tellement grave qu'il y avait « inexistence du fait ». Mais le système de protection visé à l'art. 3 du décret législatif $n^{\circ} 23 / 2015$ ne permettait pas de reconnaître à la travailleuse plaignante une protection compensatoire du préjudice réellement subi. Au contraire l'acceptation de la question de constitutionnalité permettrait - selon le Tribunal - de reconnaître à la plaignante une protection compensatoire du préjudice réellement subi, qui, dans ce cas, serait constitué par la protection spécifique prévue à l'art. 18, alinéas 4 et 7 de la Loi n 300/70 (et, à titre subsidiaire, une protection mineure ex alinéa 5). Le juge de Rome a enfin indiqué que l'employeur a, « de toute évidence, voulu profiter des avantages accordés en matière de cotisation sociale en embauchant une travailleuse dont il s'est ensuite débarrassé avec un licenciement pseudo motivé ».

La Cour a opté, après un raisonnement assez long, pour la déclaration d'illégitimité de l'art. 3, alinéa 1, dans la partie où il fixe l'indemnité pour licenciement injustifié en un « montant équivalent à deux mensualités du dernier salaire de référence pour le calcul du trattamento di fine rapporto (t.f.r.) pour chaque année de service ».

La prévision d'une indemnité augmentant en raison de la seule ancienneté de service est - selon la Cour - contraire au principe d'égalité et en violation des articles 4 (" droit au travail ») et 35 ( " protection du travail sous toutes ses formes et applications ») de la Constitution italienne, ainsi que des articles 76 et 117 (en relation avec l'art. 24 de la Charte sociale européenne révisée).

L'arrêt est « ablatif », et non " additif » dans le sens où il n'ajoute pas les critères que le juge doit respecter, éliminant juste la formule mathématique, la considérant comme insuffisante. Dès lors, on ne sait pas, à l'heure actuelle, comment appliquer cette décision aux litiges à venir.

Cependant, l'arrêt donne des indications. L'élimination des mots « montant équivalent à deux mensualités du dernier salaire (...) pour chaque année de service » s'est accompagnée, dans la motivation du jugement, par des précisions utiles. II est indiqué que le juge doit, dans le respect des limites minimales et maximales prévues par l'art. 3, al. 1, du décret $n^{\circ}$ 23/2015, pour calculer l'indemnité dédommageant le licenciement illégitime (indennità risarcitoria), tenir compte avant tout de l'ancienneté du salarié (en application de l'art. 1, al. 7, de la Loi n 184/2014), mais aussi d'autres critères prévus par l'art. 8 de la Loi n 604/66 (prévus pour le calcul de l'indemnisation dans les entreprises de moins de 16 salariés), c'est-à-dire "le nombre de salariés, la dimension de l'activité économique, ou encore le comportement et la condition de chaque partie ».

Le choix de la Cour constitutionnelle italienne d'éliminer du texte de l'art. 3, al. 1, la précision sur l'ancienneté du salarié, et d'ajouter à ce paramètre d'autres critères dans sa motivation, pourrait permettre au juge d'évaluer librement quels critères de dédommagement adopter et quelle valeur financière attribuer à chacun d'entre eux. La 
gravité de l'infraction pourra être, elle aussi, évaluée par le juge, car pouvant être tout de même déduite du " comportement (...) des parties », dans la limite du plafond de 36 mensualités.

\section{B.- LES RISQUES DE CONTRADICTION AVEC LE DROIT INTERNATIONAL}

Il existe également un risque que les droits français et italien soient contraires à certaines dispositions de droit international. En France, actuellement, certains conseils de prud'hommes ont ainsi refusé d'appliquer le barème, et, surtout, le plafond des indemnités de licenciement sans cause réelle et sérieuse car cela serait contraire à la Convention $n^{\circ} 158$ de I'OIT, et à l'article 24 de la Charte sociale européenne révisée (Cser) ${ }^{49}$. Concernant cette dernière, des réclamations collectives ont été déposées par des syndicats français ${ }^{50}$ et italiens ${ }^{51}$ devant le Comité européen des droits sociaux (Ceds) pour que celui-ci se prononce sur son éventuelle violation, à la suite de la décision sur le fond rendue par le Ceds concernant la réclamation présentée par la Finnish Society of Social Rights contre la Finlande (recl. 106/2014) $)^{52}$.

L'étude de ce contentieux nécessiterait un article à part entière. Notons cependant, en guise de conclusion, que la sécurisation des licenciements voulue par les législateurs italien et français de 2014-2015 et 2017 est gravement remise en cause, et que les mois à venir seront d'une grande incertitude juridique. En voulant limiter et plafonner les indemnisations de licenciement illégitime, les législateurs ont peut-être fabriqué des normes non plus dissuasives, mais incitatives au licenciement, risquant de se heurter à des normes constitutionnelles ou de droit international du travail. Pour dire la même chose autrement, à vouloir trop sécuriser l'employeur, on l'aurait plongé dans la totale insécurité.

\section{C - L'ENJEU SPÉCIFIQUE DU CONTRÔLE DU MOTIF ÉCONOMIQUE DE LICENCIEMENT}

Enfin, aborder la logique de sécurisation uniquement du point de vue des sanctions, et pas de celui du contrôle du motif, serait incomplet. La notion de sécurisation doit aussi être évaluée du point de vue de la justification du licenciement (notamment le licenciement économique), et de son appréciation par les juges.

49 Voir, entre-autres, CPH Lyon du 7 janvier 2019; CPH Lyon du 21 décembre 2018 ; CPH Amiens du 19 décembre 2018 ; CPH Troyes du 13 décembre 2018.

50 Recl. n 160/2018 CGT-FO c. France; recl. n 171/2018 CGT c. France ; recl. nº 174/2019 CGT YTO France c. France.

51 L'arrêt de la Cour constitutionnelle italienne n 194/2018 a été publié alors même que reste pendante la réclamation collective déposée le 26 octobre 2017 par la Confédération Générale Italienne du Travail (CGIL) c. I'Italie devant le Ceds (recl. n 158/2017). Celui-ci va se prononcer prochainement sur cette même question. En particulier la CGIL souligne dans sa réclamation la contrariété avec l'art. 24 de la CSER de la réglementation italienne de licenciement contenue dans le décret $n^{\circ} 23 / 2015$. Il convient de signaler que le gouvernement français est intervenu dans cette procédure (acte 31 mai 2018).

52 J. Mouly, «Le plafonnement des indemnités de licenciement injustifié devant le Comité européen des droits sociaux», op. cit., p. 745 ; J. Mouly, "L'indemnisation du licenciement injustifié à l'épreuve des norme supra-légales », Droit ouvrier, juillet 2018, p. 435. 
En France, la Cour de cassation a consacré, dans les années 1990, le principe d'un contrôle approfondi du motif économique ${ }^{53}$, malgré un revirement partiel effectué en 2000 par l'arrêt SAT ${ }^{54}$.

En Italie, le juge devaitégalement, traditionnellement, contrôler les motifs économiques. Cependant, depuis 2016, une nouvelle jurisprudence est applicable ${ }^{55}$, indiquant que la suppression du poste de travail, et donc le choix de gestion de l'organisation, constitue en soi un motif objectif justifié de licenciement. Cette jurisprudence exclut que dans le concept de motif justifié objectif fixé par l'art. 3 de la Loi n 604/66 (" raisons inhérentes à l'activité productive, à l'organisation du travail et à son fonctionnement régulier ») soient intégrées les raisons à l'origine de la décision organisationnelle ${ }^{56}$, ainsi que les évaluations de fond relatives à la rentabilité, opportunité, efficacité, etc., du choix de l'employeur de réorganiser sa propre structure.

Cette jurisprudence entérine donc le principe, dans les licenciements économiques italiens, d'un fort pouvoir de l'employeur de réorganiser comme il le souhaite son entreprise. On peut ainsi douter que la Cour de Cassation italienne ait réalisé une pesée raisonnable entre un droit social fondamental (celui à la stabilité au poste de travail, intégré dans le droit au travail - fixé par l'art. 4 de la Constitution italienne) et un droit économique soumis à des limites (celui du libre usage du pouvoir d'organisation et de management de l'entrepreneur, basée sur l'art. 41, alinéa 1, de la Constitution).

II y aurait ici la nécessité de revenir sur l'orientation jurisprudentielle d'origine, qui indiquait que le licenciement pour motif justifié objectif de type économique est un choix de gestion extrême (extrema ratio) en présence d'une crise économique ou de graves raisons organisationnelles.

\section{Conclusion}

Quel bilan comparé tirer de ces tentatives italiennes et françaises de sécurisation? Tant du côté de l'employeur que du salarié, dans les deux droits, le bilan est négatif.

Concernant les employeurs, la sécurité est souvent un leurre: en Italie, les plafonds d'indemnisation sont inconstitutionnels, générant une incertitude sur les litiges à venir. En France, malgré le plafonnement pour l'instant valide, un réel contrôle judiciaire, notamment

53 L'arrêt Brinon (Cass. Soc 31 mai 1956, Bull. Civ. V. 1956 n 499) indiquait que l'employeur était le seul juge du choix de licencier. Dans les années 1990, la Cour de Cassation a opéré un revirement de jurisprudence, pour contrôler de manière exhaustive les motifs économiques. Voir par exemple Cass. Soc. 2 avril 1997, n94-43165. Voir, pour une explication et une justification de cette orientation jurisprudentielle, Ph. Waquet, « Le juge et l'entreprise », Droit sociale, 1996, p. 472.

54 Cass. Ass. Plén. 8 décembre 2000, Bull. Ass. Plen. n 11. Voir notamment G. Couturier, « Licenciement économique: le choix de l'employeur et rôle du juge ", Liaisons Soc. Mag, février 2001 p. 58. La Cour de Cassation a indiqué que si les juges du fond devaient contrôler le caractère réel et sérieux du motif économique, ils ne devaient pas contrôler le choix effectué par l'employeur entre les diverses solutions possibles. En l'espèce, l'employeur envisageait initialement plusieurs types de restructurations, avec un impact plus ou moins important sur l'emploi.

55 Voir Cass. n²5201 du 7 décembre 2016. Dans le même sens Cass. 15 février 2017, n 4015 ; Cass. 20 octobre 2017, n²4882; Cass. n¹0435/2018; Cass. $n^{\circ} 16702 / 2018$.

56 Cass. 7.12.2016, n. 25201 ; Cass. 15.2.2017, n. 4015 ; Cass. 20.10.2017, n. 24882. 
des licenciements économiques, demeure. Cependant, toujours en France, le risque de contradiction des plafonds avec de droit international demeure très important à l'heure actuelle, faisant courir des risques financiers substantiels à l'employeur licenciant.

Du côté des salariés, dans les deux pays, la situation est encore pire. En Italie, l'employeur peut très facilement motiver le licenciement pour mjo de type économique, rendant toute indemnisation illusoire pour les salariés (la sanction de la réintégration ayant disparue pour les travailleurs embouchés après le 7 mars 2015). En France, pour tous les licenciements, les plafonds sont faibles, et dédommagent donc très insuffisamment les salariés injustement licenciés. L'insécurité est, à l'heure actuelle, générale, dans les deux pays, pour toutes les parties.

\section{ANDREA ALLAMPRESE}

Professeur en droit du travail, Université de Modène et Reggio Emilia

Thèmes de recherche : Droit du licenciement, droit syndical, droit social européen, détachement des travailleurs, droit de la propriété intellectuelle.

\section{Publications:}

$\sim$ A. Allamprese, « L'associazione in partecipazione con associato d'opera: un tipo contrattuale sospetto ", Lavoro e diritto, $2017, \mathrm{n}^{\circ} 2, \mathrm{p} .325$.

"La protezione dei lavoratori in una Europa in crisi : le potenzialità della Carta Sociale Europea ", E. Falletti, V. Piccone (par le soin de), Il filo delle tutele nel dedalo d'Europa, ES, Naples, 2016, p. 127.

\section{RAPHAEL DALMASSO}

Maître de conférence en droit privé, Université de Lorraine, Laboratoire de recherche IFG.

Thèmes de recherche : Droit comparé, droit des restructurations, formes modernes de travail indigne.

\section{Publications:}

R. Dalmasso, « La protection contre les formes modernes de travail indigne en France après la ratification du protocole OIT contre le travail forcé », Droit Ouvrier, 2017 p. 585.

R. Dalmasso, " Flexibiliser par la procédure: les réformes divergentes des licenciements économiques en France et en Italie ", Travail et emploi, avril-juin 2015, p. 55. " Procedural Flexibilisation of economic redundancy in France and Italy: diverging reforms with a common objective ", Travail et Emploi, 2017/5 (traduction anglaise du précédent article). 